\title{
TU/e emonownen

\section{InSb nanowire double quantum dots coupled to a superconducting microwave cavity}

\section{Citation for published version (APA):}

Wang, R., Deacon, R. S., Car, D., Bakkers, E. P. A. M., \& Ishibashi, K. (2016). InSb nanowire double quantum dots coupled to a superconducting microwave cavity. Applied Physics Letters, 108(20), [203502].

https://doi.org/10.1063/1.4950764

DOI:

10.1063/1.4950764

Document status and date:

Published: 16/05/2016

\section{Document Version:}

Publisher's PDF, also known as Version of Record (includes final page, issue and volume numbers)

\section{Please check the document version of this publication:}

- A submitted manuscript is the version of the article upon submission and before peer-review. There can be important differences between the submitted version and the official published version of record. People interested in the research are advised to contact the author for the final version of the publication, or visit the $\mathrm{DOI}$ to the publisher's website.

- The final author version and the galley proof are versions of the publication after peer review.

- The final published version features the final layout of the paper including the volume, issue and page numbers.

Link to publication

\section{General rights}

Copyright and moral rights for the publications made accessible in the public portal are retained by the authors and/or other copyright owners and it is a condition of accessing publications that users recognise and abide by the legal requirements associated with these rights.

- Users may download and print one copy of any publication from the public portal for the purpose of private study or research.

- You may not further distribute the material or use it for any profit-making activity or commercial gain

- You may freely distribute the URL identifying the publication in the public portal.

If the publication is distributed under the terms of Article 25fa of the Dutch Copyright Act, indicated by the "Taverne" license above, please follow below link for the End User Agreement:

www.tue.nl/taverne

Take down policy

If you believe that this document breaches copyright please contact us at:

openaccess@tue.nl

providing details and we will investigate your claim. 


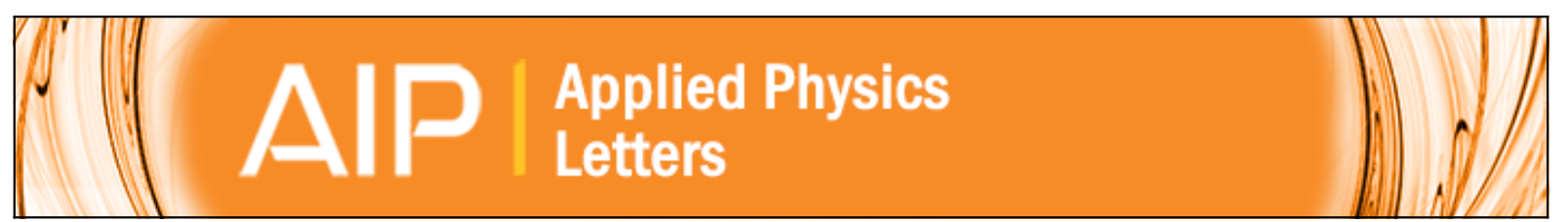

InSb nanowire double quantum dots coupled to a superconducting microwave cavity

R. Wang, R. S. Deacon, D. Car, E. P. A. M. Bakkers, and K. Ishibashi

Citation: Applied Physics Letters 108, 203502 (2016); doi: 10.1063/1.4950764

View online: http://dx.doi.org/10.1063/1.4950764

View Table of Contents: http://scitation.aip.org/content/aip/journal/apl/108/20?ver=pdfcov

Published by the AIP Publishing

\section{Articles you may be interested in}

Site-controlled formation of InAs/GaAs quantum-dot-in-nanowires for single photon emitters

Appl. Phys. Lett. 100, 263101 (2012); 10.1063/1.4731208

Counting statistics in an InAs nanowire quantum dot with a vertically coupled charge detector

Appl. Phys. Lett. 100, 072110 (2012); 10.1063/1.3687198

Tunable double dots and Kondo enhanced Andreev transport in InAs nanowires

J. Vac. Sci. Technol. B 26, 1609 (2008); 10.1116/1.2839634

Single electron pumping in InAs nanowire double quantum dots

Appl. Phys. Lett. 91, 052109 (2007); 10.1063/1.2767197

Top-gate defined double quantum dots in InAs nanowires

Appl. Phys. Lett. 89, 252106 (2006); 10.1063/1.2409625

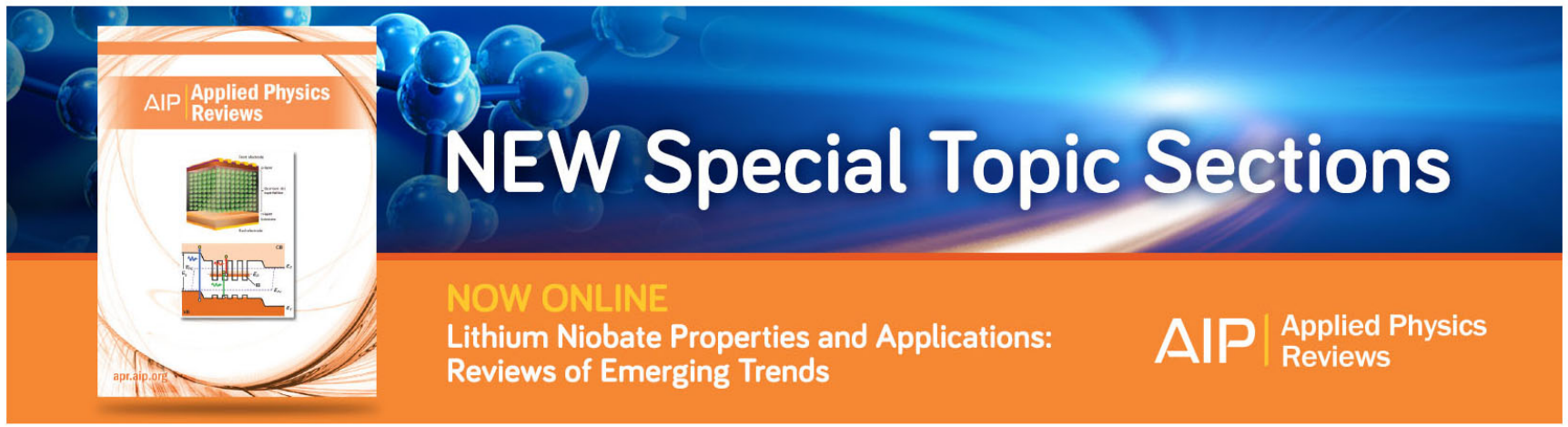




\title{
InSb nanowire double quantum dots coupled to a superconducting microwave cavity
}

\author{
R. Wang, ${ }^{1}$ R. S. Deacon, ${ }^{1,2, a)}$ D. Car, ${ }^{3}$ E. P. A. M. Bakkers, ${ }^{3,4}$ and K. Ishibashi ${ }^{1,2}$ \\ ${ }^{1}$ Advanced Device Laboratory, RIKEN, Wako, Saitama 351-0198, Japan \\ ${ }^{2}$ Center for Emergent Matter Science (CEMS), RIKEN, Wako, Saitama 351-0198, Japan \\ ${ }^{3}$ Department of Applied Physics, Eindhoven University of Technology, 5600 MB Eindhoven, The Netherlands \\ ${ }^{4}$ Kavli Institute, Quantum Transport Group, Delft University of Technology, 2628 CJ Delft, The Netherlands
}

(Received 27 February 2016; accepted 1 May 2016; published online 17 May 2016)

\begin{abstract}
By employing a micrometer precision mechanical transfer technique, we embed individual InSb nanowires into a superconducting coplanar waveguide resonator. We investigate the characteristics of a double quantum dot formed in an InSb nanowire interacting with a single mode microwave field. The charge stability diagram can be obtained from the amplitude and phase response of the resonator independently from the dc transport measurement. As the charge transits between dot-dot, or dot-lead, the change of resonator transmission is compared and the charge-cavity coupling strength is extracted to be in the magnitude of several MHz. Published by AIP Publishing.

[http://dx.doi.org/10.1063/1.4950764]
\end{abstract}

A microwave transmission line cavity can be used as a quantum bus allowing the coupling of different qubit systems on a single chip. Such coupling is already well established in superconducting qubits, ${ }^{1,2}$ and there is now interest in including other qubit systems such as nuclear spins ${ }^{3,4}$ or electron spins. ${ }^{5-12}$ A hybrid circuit quantum electrodynamics (cQED) device combining superconducting qubits with spin qubits could exploit the strengths and mitigate the weaknesses of each to create a quantum processor, for example, using the superconducting qubit for fast manipulation and the quantum dot $(\mathrm{QD})$ spin qubit as a quantum memory with long coherence time. The coupling of the electron spin to the cavity field is theoretically suggested to be achieved through spin-orbit interaction ${ }^{13}$ or the use of a slanting Zeeman field ${ }^{14}$ provided by micromagnets placed in proximity to the QD device.

Microwave cavities coupled to QD devices have been realized using gate defined GaAs QDs, ${ }^{5,6}$ InAs nanowire QDs, ${ }^{7}$ carbon nanotubes, ${ }^{8-10,12}$ and Graphene QDs. ${ }^{11}$ To date, studies mostly have focused upon the dipole charge coupling of QD and cavity. InSb nanowires exhibit large g-factor and strong spin-orbit interaction, ${ }^{15-18}$ making them a promising candidate to construct the hybrid QD-cavity architecture which can be operated in a small magnetic field to realize the potential strong spin coupling regime in which photon-charge coupling strength exceeds the cavity internal loss and the qubit decoherence rate. Here, we demonstrate a mechanical transfer technique to align single nanowires with micron accuracy onto prefabricated surface gates followed with one step lithography for the contact electrodes and the microwave cavity. This method ensures a high fabrication yield of hybrid devices in which the nanowire interacts with the strongest microwave field. A double QD is formed in the InSb nanowire with predefined local electric gates. The charge state is read out from the amplitude and phase response of resonator as well as the dc transport measurement. The frequency shift and

${ }^{\text {a)} E l e c t r o n i c ~ m a i l: ~ r u s s e l l @ r i k e n . j p ~}$ linewidth broadening of the resonator are also compared when charge transits between dot-dot, or dot-lead.

We accurately position single nanowires onto prefabricated gate electrodes using a Polyvinyl alcohol/Poly(methyl methacrylate) (PVA/PMMA) polymer bilayer technique similar to that developed to prepare stacked graphene devices. ${ }^{19}$ The process is fully detailed in the supplementary material ${ }^{20}$ but is briefly described here. First single nanowires are transferred from the growth substrate to a polymer stack of PVA/PMMA on a silicon substrate using a micromanipulator with sharp tungsten probe coated in indium. ${ }^{21}$ The PMMA layer is peeled from the PVA coated silicon substrate producing a free standing PMMA film which is aligned over the device substrate using a homemade micromanipulator alignment system. The PMMA film is brought into contact with the device substrate and heated to $150^{\circ} \mathrm{C}$ fixing the film into place. Finally, the PMMA is stripped with acetone and sample processing of contacts continues with conventional electron beam lithography and sputter deposition. We note that the transfer of single wires using the microprobe can be performed directly to prefabricated gates as reported by Kilian et al. ${ }^{21}$ however, we found a higher yield of accurate alignment from the PMMA method detailed here; as prior to heating the film, the alignment can be repeated (by pulling the film from the surface and repeating alignment) until the desired accuracy is achieved.

Our device consists of a pair of InSb nanowires used to form double QDs positioned at the ends of a half wavelength transmission line cavity as shown in Fig. 1. Nanowires are positioned across the transmission line gap with source contacted to the signal line and drain connected to the resonator ground plane. The prefabricated gate electrodes have a pitch of $100 \mathrm{~nm}$ and are covered with a nominal $30 \mathrm{~nm}$ thick PECVD grown $\mathrm{Si}_{3} \mathrm{~N}_{4}$ dielectric layer. A dc bias is supplied to the nanowire using an inductor placed at the center of the cavity to avoid the microwave leakage from the dc port (and hence maintain the high quality of the resonator). In this study, we focus on the detection of the electron state and the 


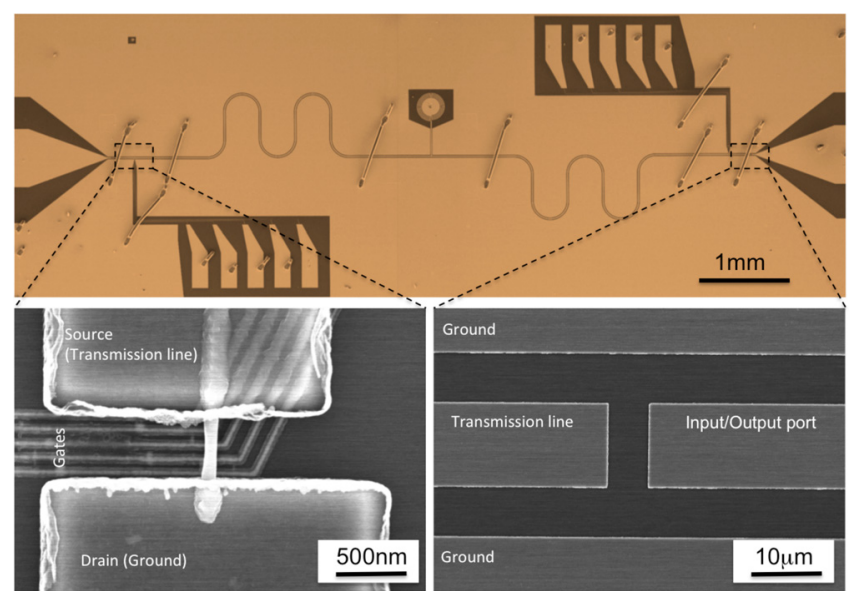

FIG. 1. Scanning electron microscope (SEM) image in false color of a coplanar superconducting micro-cavity made of $150 \mathrm{~nm}$ thick $\mathrm{Nb}$ film on a $8 \times$ $4 \mathrm{~mm}^{2}$ silicon chip. The central conductor is $10 \mu \mathrm{m}$ wide separated from the ground plane with a gap of $6 \mu \mathrm{m}$. Lower panels are the magnified SEM pictures of nanowire double QD bridging between transmission line and ground plane and the coupling capacitor at the ends of resonator, respectively.

charge dipole coupling of only one nanowire, and the other is pinched off by applying negative voltage to all gates. Measurements are performed in a $\mathrm{He}^{3}-\mathrm{He}^{4}$ dilution refrigerator with a base temperature of $\sim 30 \mathrm{mK}$. The cavity transmission is measured using a cryogenic HEMT amplifier, a chain of room temperature amplifiers, and a vector network analyzer. ${ }^{20}$

The double QD is formed by introducing local potential barriers along the InSb nanowires with gates nearby the source/drain and in the center (denoted as $\mathrm{V}_{S G}, \mathrm{~V}_{D G}, \mathrm{~V}_{M G}$, respectively). The barriers determine the confinement of charges in each dot, and the middle gate is used to tune the interdot coupling rate $\left(t_{c}\right)$. The chemical potential of each $\mathrm{QD}$ is controlled by plunger gates $\left(\mathrm{V}_{p 1}, \mathrm{~V}_{p 2}\right)$. During the $\mathrm{RF}$ measurement, the dc bias is set to zero, and the RF power at the input port of the resonator is $-86 \mathrm{dBm}$ (corresponding to approximately $1000 \mathrm{~s}$ of cavity photons). The transmission power spectrum of the bare resonator (when QDs are in the coulomb blockade) shows a Lorentzian peak (blue line, Fig. 2(a)). The single mode microwave frequency is at $f_{0}=6.0749 \mathrm{GHz}$ with a linewidth of $\kappa / 2 \pi \sim 750 \mathrm{KHz}$, corresponding to a loaded quality (Q) factor of 8000 which is limited by the cavity internal loss and the input/output capacitive coupling. The averaged life time $(1 / \kappa)$ of photons trapped in the cavity exceeds $200 \mathrm{~ns}$. In comparison, the resonance of the microwave cavity shows a damped peak when QDs are in the charge degeneracy regime (red line, Fig. 2(a)), while the phase signal (red line, Fig. 2(b)) displays both a decrease of central frequency and Q-factor. These results indicate that the resonator transmission is sensitive to the charge states of the QDs. Figs. 2(c) and 2(d) show the variation of amplitude and phase signal, respectively, of the resonator driven at the bare load frequency, $f_{0}$. Each diagram displays a similar honeycomb structure as a function of $\mathrm{V}_{p 1}$ and $\mathrm{V}_{p 2}$, indicating a readout of the charge states of the QDs in good agreement with previous reports on other QDcavity systems. ${ }^{8,10}$

In Figs. 3(a) and 3(b), we present charge stability diagrams plotted as the variation of amplitude and phase with
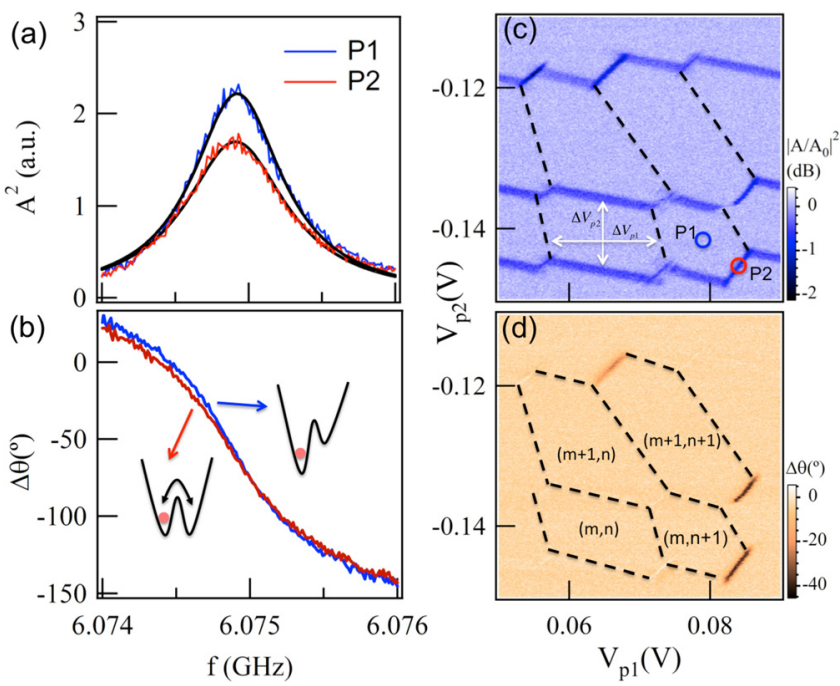

FIG. 2. Measured amplitude (a) and phase (b) of the transmission spectra of the resonator at points P1 (blue) and P2 (red) indicated in (c). The black solid lines are a best fit to a Lorentzian function. (c) The amplitude and (d) phase variation of the transmission signal driven at the bare resonator frequency as a function of the two plunger gate voltages. A background obtained with the double QD in the Coulomb blockade regime is subtracted in both plots. $\Delta V_{p 1(p 2)}$ measures the plunger gate voltage difference to populate or deplete single electron in each QD as indicated by the white double arrows in (c) between the parallel edges of the hexagonal pattern. Gate voltages $\mathrm{V}_{S G}, \mathrm{~V}_{D G}$, and $\mathrm{V}_{M G}$ are $0.42 \mathrm{~V}, 1.0 \mathrm{~V}$, and $0.5 \mathrm{~V}$, respectively.

the interdot tunnelling rate reduced relative to that in Fig. 2 by applying a lower barrier gate voltage, $V_{M G}=0.47 \mathrm{~V}$. The charging energy of each QD and the capacitive lever-arm $\left(\alpha_{p 1}, \alpha_{p 2}\right)$ of each plunger gate can be extracted from the dc-transport measurements ${ }^{22}$ (see supplementary material ${ }^{20}$ ). The capacitive coupling between the two QDs varies with the charge state of QD1 as indicated by the change of the length of the interdot tunnelling lines (marked by the yellow
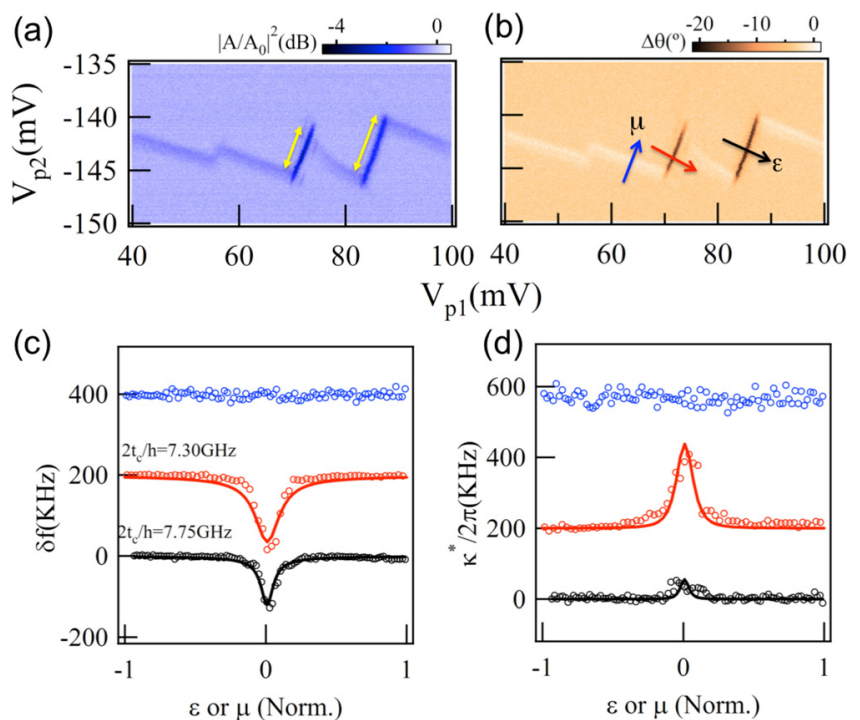

FIG. 3. (a) Amplitude and (b) phase variation of the transmission signal at the bare resonator frequency as a function of the two plunger gate voltages. The gate bias $V_{M G}=0.47 \mathrm{~V}$ with other barrier gate voltages fixed at VSG $=0.42 \mathrm{~V}$ and $\mathrm{V} D G=1.0 \mathrm{~V}$. (c) Frequency shift and (d) linewidth change of the resonator as a function of energy detuning $\varepsilon$ between the two QDs and chemical potential $\mu$ with respect to the tunnelling resonance level of QD2, respectively. The energy axes are normalized in both plots and the scale factors are $0.4 \mathrm{meV}$ (black), $0.17 \mathrm{meV}$ (red), and $0.3 \mathrm{meV}$ (blue), respectively. The frequency shift and linewidth are vertically offset for clarity. 
double arrows in Fig. 3(a)). The transmission spectrum is investigated using two sets of vector gate sweeps as illustrated with arrows in Fig. 3(b). First, the plunger gates are swept across one QD charging line (blue arrow in Fig. 3(b)), while the tunnelling rate between the QD and the adjacent reservoir changes as the chemical potential of the QD is shifted by $\mu_{1(2)}=\alpha_{p 1(2)} \Delta \mathrm{V}_{p 1(2)}$. The black and red arrows perpendicular to the interdot lines indicate a set of gate sweeps for which the energy detuning between the QDs is tuned by $\varepsilon=\mu_{1}-\mu_{2}$, while the mean chemical potential $\left(\mu_{\text {mean }}=\mu_{1}+\mu_{2}\right)$ is fixed. All the resonator spectra measured as a function of probe frequency show a Lorentzian shape (see supplementary material ${ }^{20}$ ). The frequency shift $(\delta \mathrm{f})$ and linewidth variation $\left(\kappa^{*} / 2 \pi\right)$ as a function of $\varepsilon$ and $\mu$ are plotted in Figs. 3(c) and 3(d), respectively. The energy scale is normalized, and the results are vertically offset for clarity in both plots (details shown in figure caption). A pronounced negative frequency shift is observed when the gate voltage is set on the interdot tunnelling line where $\varepsilon=0$ as shown by black and red open dots in Fig. 3(c). The coupled double QD can be interpreted as a charge qubit encoded by the bonding/ antibonding states. ${ }^{22}$ The double QD embedded in the microcavity is capacitively coupled to the microwave photons with a Jaynes-Cummings type interaction. ${ }^{1,23}$ Taking into account the charge qubit decoherence rate $\gamma_{t o t}=\gamma_{e} / 2+\gamma_{\phi}$ $\left(\gamma_{e}\right.$ and $\gamma_{\phi}$ are the energy relaxation and dephasing rate, respectively), ${ }^{7,24}$ the strength of photon-qubit interaction is characterized by the double QD susceptibility to the microwave field ${ }^{7,12}$

$$
\chi=\frac{g_{c}}{i\left(\gamma_{t o t} / 2\right)-\triangle}
$$

where $g_{c}=g_{0} \frac{2 t_{c}}{\Omega}$ is the effective dipole-photon coupling strength. $\triangle=\Omega-h f_{0}$ is the double QD-cavity detuning, and $\Omega=\sqrt{\varepsilon^{2}+4 t_{c}^{2}}$ and $t_{c}$ are the interdot tunnelling rate. The central frequency of the resonator is shifted by $\operatorname{Re}\left(g_{c} \chi\right)$, and the linewidth is broadened by $2 \operatorname{Im}\left(g_{c} \chi\right)$. The observed negative frequency shift indicates that the minimal interdot tunnelling coupling strength of charge qubit, $2 t_{c}=\Omega(\varepsilon=0)$, is larger than the resonator photon energy according to Eq. (1). With a coupling strength of $g_{0} / 2 \pi=14 \mathrm{MHz}$, the best fit to all data gives the interdot coupling rate around $7 \mathrm{GHz}$, which is comparable with previous reports on other double QD systems. ${ }^{5,7,10}$ It is worthwhile to point out that as $t_{c}$ becomes smaller, we observe not only a larger negative frequency shift (Fig. 3(c)) but also a clear linewidth broadening (Fig. 3(d)) due to the charge qubit decoherence. Assuming a typical energy relaxation rate of $100 \mathrm{MHz}$ of a charge dipole qubit, ${ }^{24-26}$ our result allows estimation of the charge imhomogeneous dephasing rate $\gamma_{\phi}=1 \sim 4 \mathrm{GHz}$, which significantly exceeds the coupling strength and is likely attributed to slow charge noise. . $^{57}$

In contrast to the case of interdot tunnelling, the central frequency and linewidth of the resonator show negligible variation when electrons transit between one QD and reservoir (blue open dots in Figs. 3(c) and 3(d)). To interpret the results, the double QD is commonly treated as a parallel RC circuit connecting to a RF sensor as shown in Fig. 4(d). The
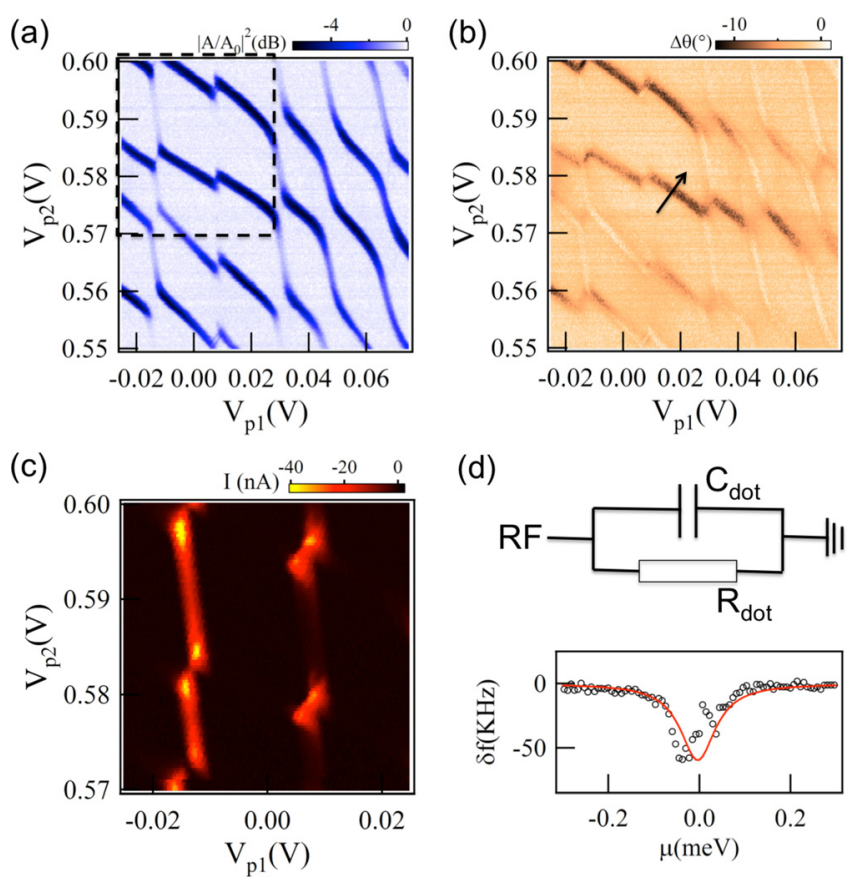

FIG. 4. (a) Amplitude and (b) phase variation of the transmission signal at the bare resonator frequency. (c) DC-transport measurement obtained with a bias of $300 \mu \mathrm{V}$ corresponding to the dashed frame in (a) with no rf power applied. (d) Scheme of RC circuit equivalent to double QD and the measured frequency shift (black open dot) when gates sweep across a charging line of one QD as indicated by the arrow in (b). The red solid line is a fitting with a scattering matrix approach. ${ }^{28}$

change of amplitude and phase of the RF signal responds to the dynamical admittance of double $\mathrm{QD}^{8,29,30}$

$$
Y(\omega)=R_{d o t}^{-1}+j \omega C_{d o t},
$$

where $R_{d o t}$ and $C_{d o t}$ are the effective resistance and capacitance of the double QD, which are determined by the capacitive coupling strength $\left(\alpha_{1(2)}\right)$ between each QD and the RF line as well as the ratio of charge transition rate over the microwave frequency $\left(f_{0} / \tau\right)$ described by a scattering matrix model. ${ }^{28,29,31,32}$ This model has been applied to sensors using the reflectometry technique for fast readout of the charge or spin state of various single or double QD devices. ${ }^{7,10,33-35}$ The charging lines on QD2 (Figs. 2(c) and 3(a)) are clear because the transmission line is close to QD2 with a large capacitive coupling strength while QD1, being further away, has a weaker coupling. The change in phase shift signal is always negligible perhaps due to the charge transition rate between QD and transmission line being low. Previous studies have demonstrated that the phase response will be enhanced by increasing the tunnelling rate at the contact. ${ }^{10,29}$ Figs. 4(a) and 4(b) show the amplitude and phase response of the resonator when a large $\mathrm{V}_{p 2}$ is applied, and now, the charging lines of both QDs are observed. In contrast, in the dc transport presented in Fig. 4(c), the current at the triple points and the charging lines of QD1 are more prominent because the tunnelling rate through QD2 is now comparatively high. The results indicate that the mechanisms of dc transport and RF detection are different. Note that dc current carriers need to transport through both QDs, while for RF detection, the charges only need transit between a lead and 
either QD. The effective capacitor $\mathrm{C}_{d o t}$ causes a shift of frequency $\delta \mathrm{f} \approx-C_{\text {dot }} f_{0} /\left(2 C_{\text {res }}\right)$, where $C_{\text {res }}=0.8 \mathrm{pF}$ is the resonator capacitor. ${ }^{36}$ As the plunger gates sweep across one charging line of QD2 (indicated by the arrow in Fig. 4(b)), the tunnelling rate between QD2 and reservoir is tuned and the frequency displays a negative shift (Fig. 4(d)). With a parameter $\alpha=0.03$, the fitting gives $\mathrm{C}_{d o t}$ of approximately $30 \mathrm{aF}$ and the tunnelling rate between QD2 and lead is estimated as approximately $8 \mathrm{GHz}$. We also observe slight positive phase shifts on some of the charging lines as shown in Fig. 4(b), which indicates the crossover of capacitive and inductive response as the charge state of the QDs changes. A plausible mechanism is that tunnelling barrier at the contacts is modified as the gate voltage sweeps. As the tunneling rate decreases lower than the microwave frequency, the dwell time of the electron in the QD increases beyond the microwave period. The electron cannot follow the field drive any more, presenting an inductive behaviour. Our observation is consistent with the work reported by Frey et al. who demonstrated the inductive response of a GaAs double QD coupled to a microwave cavity. ${ }^{29}$ Most of the charging lines of QD1 in Fig. 4 are slightly brighter, indicating the inductive behaviour while most of the charging lines of QD2 show negative phase (or frequency) shift attributed to the capacitive response. These results are also consistent with the indications from dc transport that the QD2 exhibits larger electron transition rate with the lead.

In summary, we embed individual InSb nanowires into a coplanar waveguide microcavity by developing a mechanical transfer technique, which is applicable to other selfassembled nanowires. The microwave resonator is exploited as a nondemolition sensor to read out the charge state of double QDs formed in the nanowires. The charge dipole in the double QD interacting with microwave photons and the dynamic admittance of the QD circuit is analyzed and interpreted with different mechanisms. Such hybrid architecture will be used as a platform for future research on the spincavity coupling.

We thank Y. Yamazaki for preliminary device preparation and T. Yamaguchi for result discussion. The work was partially supported by Grants-in-Aid for Scientific Research (A) (No.15H02015) and The Netherlands Organization for Scientific Research (NWO VICI and NWO groot). R.W. acknowledges financial support from the foreign postdoctoral researcher (FPR) program of Riken and R.S.D. acknowledges support from Grants-in-Aid for Young Scientists B (No. 26790008).

\footnotetext{
${ }^{1}$ A. Blais, R.-S. Huang, A. Wallraff, S. M. Girvin, and R. J. Schoelkopf, Phys. Rev. A 69, 062320 (2004).

${ }^{2}$ A. Wallraff, D. I. Schuster, A. Blais, L. Frunzio, R.-S. Huang, J. Majer, S. Kumar, S. M. Girvin, and R. J. Schoelkopf, Nature 431, 162 (2004).

${ }^{3}$ Y. Kubo, F. R. Ong, P. Bertet, D. Vion, V. Jacques, D. Zheng, A. Dréau, J.-F. Roch, A. Auffeves, F. Jelezko, J. Wrachtrup, M. F. Barthe, P. Bergonzo, and D. Esteve, Phys. Rev. Lett. 105, 140502 (2010).

${ }^{4}$ P. Rabl, D. DeMille, J. M. Doyle, M. D. Lukin, R. J. Schoelkopf, and P. Zoller, Phys. Rev. Lett. 97, 033003 (2006).
}

${ }^{5}$ T. Frey, P. J. Leek, M. Beck, A. Blais, T. Ihn, K. Ensslin, and A. Wallraff, Phys. Rev. Lett. 108, 046807 (2012).

${ }^{6}$ S. Toida, H. Nakajima, and S. Komiyama, Phys. Rev. Lett. 110, 066802 (2013).

${ }^{7}$ K. D. Petersson, L. W. McFaul, M. D. Schroer, M. Jung, J. M. Taylor, A. A. Houck, and J. R. Petta, Nature 490, 380 (2012).

${ }^{8}$ M. R. Delbecq, V. Schmitt, F. D. Parmentier, N. Roch, J. J. Viennot, G. Fève, B. Huard, C. Mora, A. Cottet, and T. Kontos, Phys. Rev. Lett. 107, 256804 (2011).

${ }^{9}$ M. R. Delbecq, L. E. Bruhat, J. J. Viennot, D. Datta, A. Cottet, and T. Kontos, Nat. Commun. 4, 1400 (2013).

${ }^{10}$ V. Ranjan, G. Puebla-Hellmann, M. Jung, T. Hasler, A. Nunnenkamp, M. Muoth, C. Hierold, A. Wallraff, and C. Schönenberger, Nat. Commun. 6, 7165 (2015).

${ }^{11}$ G.-W. Deng, D. Wei, J. Johansson, M.-L. Zhang, S.-X. Li, H.-O. Li, G. Cao, M. Xiao, T. Tu, G.-C. Guo, H.-W. Jiang, F. Nori, and G.-P. Guo, Phys. Rev. Lett. 115, 126804 (2015).

${ }^{12}$ J. J. Viennot, M. Dartiailh, A. Cottet, and T. Kontos, Science 349, 408 (2015).

${ }^{13}$ M. Trif, N. V. Golovach, and D. Loss, Phys. Rev. B 77, 045434 (2008).

${ }^{14}$ X. Hu, Y. X. Liu, and F. Nori, Phys. Rev. B 86, 035314 (2012).

${ }^{15}$ V. S. Pribiag, S. Nadj-Perge, S. M. Frolov, J. W. G. van den Berg, I. van Weperen, S. R. Plissard, E. P. A. M. Bakkers, and L. P. Kouwenhoven, Nat. Nanotechnol. 8, 170 (2013).

${ }^{16}$ S. Nadj-Perge, V. S. P. W. G. van den Berg, K. Zuo, S. R. Plissard, E. P. A. Bakkers, S. M. Frolov, and L. P. Kouwenhoven, Phys. Rev. Lett. 108, 166801 (2012).

${ }^{17}$ J. W. G. van den Berg, S. Nadj-Perge, V. S. Pribiag, S. R. P. E. P. A. M. Bakkers, S. M. Frolov, and L. P. Kouwenhoven, Phys. Rev. Lett. 110, 066806 (2013).

${ }^{18}$ I. van Weperen, B. Tarasinski, D. Eeltink, V. Pribiag, S. R. Plissard, E. P. A. M. Bakkers, L. P. Kouwenhoven, and M. Wimmer, Phys. Rev. B 91, 201413 (2015).

${ }^{19}$ T. Taychatanapat, K. Watanabe, T. Taniguchi, and P. Jarillo-Herrero, Nat. Phys. 7, 621 (2011).

${ }^{20}$ See supplementary material at http://dx.doi.org/10.1063/1.4950764 for additional fabrication details and measurements.

${ }^{21}$ K. Flöhr, M. Liebmann, K. Sladek, H. Y. Günel, R. Frielinghaus, F. Haas, C. Meyer, H. Hardtdegen, T. Schäpers, D. Grützmacher, and M. Morgenstern, Rev. Sci. Instrum. 82, 113705 (2011).

${ }^{22}$ W. van der Wiel, S. D. Franceschi, J. Elzerman, T. Fujisawa, S. Tarucha, and L. Kouwenhoven, Rev. Mod. Phys. 75, 1 (2002).

${ }^{23}$ L. Childress, A. S. Sørensen, and M. D. Lukin, Phys. Rev. A 69, 042302 (2004).

${ }^{24}$ J. R. Petta, A. C. Johnson, C. M. Marcus, M. P. Hanson, and A. C. Gossard, Phys. Rev. Lett. 93, 186802 (2004).

${ }^{25}$ T. Fujisawa, T. H. Oosterkamp, W. G. van der Wiel, B. W. Broer, R. Aguado, S. Tarucha, and L. P. Kouwenhoven, Science 282, 932 (1998).

${ }^{26}$ T. Hayashi, T. Fujisawa, H. D. Cheong, Y. H. Jeong, and Y. Hirayama, Phys. Rev. Lett. 91, 226804 (2003).

${ }^{27}$ F. Forster, G. Petersen, S. Manus, P. Hänggi, D. Schuh, W. Wegscheider, S. Kohler, and S. Ludwig, Phys. Rev. Lett. 112, 116803 (2014).

${ }^{28}$ A. Prêtre, H. Thomas, and M. Büttiker, Phys. Rev. B 54, 8130 (1996).

${ }^{29}$ T. Frey, P. J. Leek, M. Beck, J. Faist, A. Wallraff, K. Ensslin, T. Ihn, and M. Büttiker, Phys. Rev. B 86, 115303 (2012).

${ }^{30}$ S. J. Chorley, J. Wabnig, Z. V. Renfold-Fitch, K. D. Petersson, J. Frake, C. G. Smith, and M. R. Buitelaar, Phys. Rev. Lett. 108, 036802 (2012).

${ }^{31}$ A. Cottet, C. Mora, and T. Kontos, Phys. Rev. B 83, 121311(R) (2011).

${ }^{32}$ M. G. House, T. Kobayashi, B. Weber, S. J. Hile, T. F. Watson, J. van der Heijden, S. Rogge, and M. Y. Simmons, Nat. Commun. 6, 8848 (2015).

${ }^{33}$ A. P. Higginbotham, T. W. Larsen, J. Yao, H. Yan, C. M. Lieber, C. M. Marcus, and F. Kuemmeth, Nano Lett. 14, 3582 (2014).

${ }^{34}$ D. J. Reilly, C. M. Marcus, M. P. Hanson, and A. C. Gossard, Appl. Phys. Lett. 91, 162101 (2007).

${ }^{35}$ M. Jung, M. D. Schroer, K. D. Petersson, and J. R. Petta, Appl. Phys. Lett. 100, 253508 (2012).

${ }^{36}$ M. Göppl, A. Fragner, M. Baur, R. Bianchetti, S. Filipp, J. M. Fink, P. J. Leek, G. Puebla, L. Steffen, and A. Wallraff, J. App. Phys. 104, 113904 (2008). 